\title{
La influencia de los espacios para el desarrollo del proceso de enseñanza/aprendizaje en educación física: una perspectiva autoetnográfica \\ The influence of spaces for the development of the teaching/learning process in physical education: an autoethnographic perspective \\ *Gustavo González-Calvo, *Lucio Martínez-Álvarez, **David Hortigüela-Alcalá \\ *Universidad de Valladolid,**Universidad de Burgos
}

\begin{abstract}
Resumen. Uno de los elementos que más condicionan la práctica pedagógica, dentro de la Educación Física, tiene que ver con los espacios en que se desarrolla la tarea docente. En este artículo se expone el proceso reflexivo de un educador novel en el que se van revisando sus principios, propósitos y consecuencias de sus acciones en relación con la creación de espacios de enseñanza/aprendizaje seguros y educativos para todo el alumnado. El artículo adopta una metodología cualitativa, en forma de autoetnografía, para conocer la influencia que ejercen los espacios en los que se desarrolla la Educación Física en la concepción y afrontamiento de la materia. Los resultados reflejan cómo una de las mayores preocupaciones del docente tiene que ver con adaptar los espacios y los contextos en que se desarrollan las lecciones para reducir las contingencias.

Palabras clave: Autoetnografía; diario de clases; investigación cualitativa; identidad profesional.
\end{abstract}

\begin{abstract}
One of the elements that most influence the pedagogical practice, within the Physical Education, has to do with the spaces in which the teaching task is developed. This article exposes the reflexive process of a novel educator in which he reviews his principles, purposes and consequences of their actions in relation to the creation of safe teaching and learning spaces for all students. The article adopts a qualitative methodology, in the form of autoethnography, to know the influence exerted by the spaces in which Physical Education develops in the conception and confrontation of the subject. The results reflect how one of the main concerns of the teacher has to do with adapting the spaces and contexts in which the lessons are developed to reduce contingencies.
\end{abstract}

Keywords: Autoethnography; teaching diary; qualitative research; professional identity.

\section{Introducción}

Dentro del área de Educación Física, alumnado, profesorado y contexto son los tres pilares básicos sobre los que se configura la materia (Öhman \& Quennerstedt, 2017). Una vez que el docente tenga un dominio y comprensión apropiados de estos tres aspectos, aumentarán las prácticas pedagógicas que tengan un verdadero sentido de aprendizaje (Garret \& Wrench, 2014). Si bien la literatura refleja diversidad de experiencias en torno a la interacción existente entre docente y discente, son escasas las que se centran específicamente en cómo percibe e interactúa el profesorado de Educación Física con los espacios y materiales de los que dispone. Esto, que un principio puede parecer de escasa relevancia, aporta mucha luz e información a la manera en la que concibe su práctica y crea así su identidad profesional (Chong, Low, \& Goh, 2011; Dervent, 2015; González Calvo, Barbero González, Bores Calle, \& Martínez Álvarez, 2014). Además, puede encontrarse estrechamente relacionado con la metodología empleada en el aula y con la forma de organizar y temporalizar los contenidos. Un espacio puede ser, entre otros: abierto, cerrado, frío, caluroso, amplio, reducido, nuevo o deteriorado, y la percepción y visualización de práctica por parte del docente puede conllevar un tipo u otro de uso (MacPhail \& Halbert, 2010). Algo similar sucede con los materiales, pues sus características, su uso, si son o no autoconstruidos o la relación del alumnado con el mismo, determinan la forma en la que se aborda la materia.

Un espacio puede ser concebido de una forma muy distinta por diferentes docentes, derivando en la generación y acotación de subespacios que definan prácticas pedagógicas bien diferenciadas. Ocurre algo similar respecto a las condiciones de seguridad y de riesgo que emanan de la práctica de actividad física en el aula. Aquí hay que destacar dos términos diferentes y comúnmente confundidos, el riesgo objetivo y el subjetivo. Mientras que el riesgo objetivo es real, el subjetivo únicamente atiende a la percepción del sujeto (McCoy, Esslinger, \& Baghurst, 2017). Esto se observa claramente en actividades físicas en el medio natural, como por ejemplo el rápel, donde el miedo a realizarlo en muchos casos es alto a pesar de que las condiciones de seguridad sean mucho más elevadas que en muchos deportes colectivos. Pero más allá

Fecha recepción: 15-01-18. Fecha de aceptación: 05-03-18 Gustavo González-Calvo

gustavogonzalezcalvo@gmail.com del riesgo real, el riesgo que perciba el docente en al usar los espacios y el material en las clases de Educación Física se configura como un claro determinante de los contenidos aplicados (Merrie, Shewmake, \& Calleja, 2016).

Estos aspectos están muy vinculados a la priorización de los ejes temáticos que el docente establece en su programación de aula. En función de cómo el docente asuma las posibilidades pedagógicas que tiene el espacio de práctica y los materiales a utilizar, su rol será distinto en el aula, variará la estructura de la sesión, la implicación estudiantil en las tareas y su nivel de autonomía y relación con sus iguales en clase (Lund, 2013). Afirma este autor que los espacios y materiales además de la relación entre docentey discente, determinan la imagen y la tipología del centro educativo. Además, los espacios tienen una evidente influencia en el modo en que el docente hace sus elecciones, al tiempo que el modo en que el educador «mira» esos elementos va a estar condicionada por su formación, conocimientos, experiencias y vivencias previas (González Calvo, 2013b; González Calvo \& Barba, 2013). En este sentido, la Educación Física tiene un vínculo muy particular con el contexto en que se desarrolla, tanto por las posibilidades que ofrece dicho contexto como por los significados que se ha construido en torno aél.

Si bien la literatura ha abordado esta información desde un plano más teórico (Blández Ángel, 1995; Chappell, 2006), parece necesario conocer cómo esta situación es percibida desde la práctica profesional docente, teniendo en cuenta sus miedos, inseguridades y reflexiones. La forma en la que un docente de EF se enfrenta a su realidad y da respuesta a las situaciones con las que se encuentra, permite realizar un análisis más profundo e interno de la concepción de su praxis curricular (Stolz \& Kirk, 2015). Esto se vincula directamente con la utilidad que el docente otorgue a los espacios y los materiales. Cobra más relevancia si esos pensamientos y reflexiones se registran a lo largo del tiempo, al permitir ver cómo afronta y concibe su profesión, dando alternativas de uso a los materiales según avanza en su desarrollo profesional. De este modo, y a través del registro autobiográfico, se puede analizar con detalle de quémanera evolucionan los miedos y las inseguridades iniciales del docente de Educación Física (ver, por ejemplo, González-Calvo \& Arias-Carballal, 2017; González Calvo, 2013a, 2013b; González Calvo \& Becerril González, 2013).

El principal aporte del presente estudio es comprender las interacciones que tienen el espacio y los materiales con el desarrollo profesional del docente. Esta interacción, tal y como aquí aparece refle- 
jada, tiene una doble dirección: los espacios y materiales influyen en la práctica pedagógica (permiten unas opciones y no permiten otras) y, al tiempo, su conocimiento y disposición posibilitan que se vea en los espacios y materiales un rango de posibilidades pedagógicas (el conocimiento pedagógico permite vislumbrar un conjunto de posibilidades u otro). Podríamos afirmar que el rango de 'lo posible' está configurado tanto por lo que 'objetivamente' permite el espacio y materiales, como por lo que 'subjetivamente' es percibido dentro de un rango de lo posible. Para poder comprender la subjetividad de los contextos en que se desarrolla la Educación Física, se ha optado por un análisis autoetnográfico que permita dar voz e identidad al profesor (Chang, 2013; Holman Jones, Adams, \& Ellis, 2013), agente fundamental sobre el que gira la acción educativa y favorezca la comprensión personal y contextual del fenómeno de estudio.

\section{Metodología}

\section{Participante y recogida de datos}

Nuestra investigación se centra en la entrada profesional de un estudiante en formación inicial de Educación Física y en sus cinco primeros años de profesión como docente de Educación Física. A lo largo de su recorrido profesional, ha desempeñado su labor en las etapas de Educación Primaria, Enseñanza Secundaria y en la Universidad.

Ya en su periodo de formación inicial se va configurando como un docente reflexivo e iniciándose en la escritura de textos autobiográficos como metodología analítica que posibilita entender y mejorar su práctica.

Una vez finalizados los estudios como maestro especialista en Educación Física, cursó la licenciatura en Ciencias de laActividad Física y el Deporte y, posteriormente, su tesis doctoral, estando siempre presente un interés por dedicarse al mundo de la educación.

Tras aprobar las oposiciones al cuerpo de maestros de EF, compagina la enseñanza de la EF en la etapa de Educación Primaria con la enseñanza universitaria desde el año 2011.

En esta investigación se adoptó un enfoque fenomenológico, narrativo, reflexivo y autobiográfico para adentrarse en los mundos personales del participante (Delle Fave, 2009; Pérez-Samaniego, Devís-Devís, Smith, \& Sparkes, 2011). Dicho enfoque coloca en primer plano al sujeto, haciendo valer su voz como herramienta de investigación y de interpretación. El docente, a través de un profundo proceso de reflexión e introspección, puede enfrentarse a sus experiencias y extraer de ellas significados de forma activa.

Los datos partieron de una fuente fenomenológica principal: diversos diarios de campo, en los que se fueron recogiendo experiencias y reflexiones en relación a los objetivos de la investigación.

Diferentes autores (González Calvo \& Barba, 2014; González Calvo \& Martínez Álvarez, 2009; Liston \& Zeichner, 2003; Surbeck, Han, \& Moyer, 1991; Zabalza Beraza, 2008) resaltan que los diarios constituyen una herramienta útil y estimuladora de procesos críticos y reflexivos, ya que ayudan a ampliar el pensamiento acerca de los acontecimientos que tienen lugar en el aula y posibilitan que los docentes profundicen en sus propias acciones e ideas sobre los procesos de enseñanza/aprendizaje.

La primera experiencia con el diario de clases del investigadorparticipante surgió en su etapa de formación inicial como maestro especialista de EF, en el año 2005, lo que le permitió comprobar su utilidad como instrumento de formación y de investigación, al posibilitar la recogida de datos significativos, la reflexión sobre los mismos, su análisis y sistematización. Desde entonces, el investigador-participante continuó empleando sistemáticamente este instrumento de recogida de información, contando para este estudio con los diarios de cuatro años: (a) como estudiante de formación inicial del título de maestro especialista en Educación Física, correspondiente al año 2005; (b) como docente de Educación Primaria de los cursos 2009-2010, 2011-2012 y 20122013; y (c) como profesor de Enseñanza Secundaria durante el curso 2010-2011.

\section{Análisis de los datos}

Los diarios de clase constituyeron «prácticas narrativas» (De Fina \& Georgakopoulou, 2008) que, en sí, condujeron a «actos de significado» (Bruner, 1990) sobre la identidad profesional del docente como un narrador de sí mismo y de su propia experiencia (Smith \& Sparkes, 2008). Por otra parte, ayudaron al investigador-participante, en su análisis, a establecer una triangulación y coherencia en las relaciones teórico-prácticas entre ambas fuentes de información (ver Gergen, 1997; Goffman, 1981; Gubrium \& Holstein, 1998, 2009; Lieblich, TuvalMashiach, \& Zilber, 1998), lo cual también aumentó la credibilidad y transferibilidad del estudio (Denzin, 1989; Guba \& Lincoln, 2005; Riessman, 2008; Shenton, 2004).

Conviene destacar que esta investigación es fruto de un estudio más amplio, en el que la recogida y categorización de los datos se realizó siguiendo un procedimiento mixto inductivo-deductivo. Inductivo, pues la categorización se realizaba a medida que se examinaban los datos, aflorando diferentes categorías a medida que se iba profundizando en los mismos. Deductivo, pues ya se habían establecido previamente una serie de ideas sobre las que posteriormente se llevaría a cabo la categorización. Entre las categorías establecidas, destacan las siguientes: (a) Relaciones que establece el docente novel con los demás miembros de la comunidad educativa; (b) autoestima y pensamientos del docente (evolución del yo docente, temores y sentimientos de incompetencia como profesional, sentimientos de competencia profesional como educador según la perspectiva propia y la de los demás); (c) motivaciones laborales (sentimientos de compromiso o de desilusión hacia la profesión, relaciones entre la vida personal y profesional, falta de consideración hacia la profesión docente, estatus de la Educación Física, dilemas del docente); $\mathrm{y}$ (d) reajuste del proceso de enseñanza/aprendizaje (dificultades e implicaciones que surgen respecto a las tareas propuestas, condiciones particulares del espacio donde se llevan a cabo las lecciones de la asignatura, influencia de los materiales de trabajo, seguridad y gestión de los riesgos en la práctica pedagógica).

\section{Resultados}

En el apartado de resultados vamos a mostrar, desde una perspectiva autoetnográfica, la relación y adaptación que hace de los contenidos de la asignatura de Educación Física. Los resultados abordan cuatro temas: (a) Las instalaciones y los espacios como condicionantes de la práctica pedagógica; (b) la construcción de un ambiente seguro sobre el que desarrollar la práctica pedagógica; (c) la influencia del diseño y cuidado de las infraestructuras en el desarrollo de la Educación Física; y (d) la influencia de los espacios y las infraestructuras en el desarrollo de la identidad profesional docente.

Las instalaciones y los espacios como condicionantes de la práctica pedagógica

Desde su etapa como maestro en prácticas, era consciente de que «el modelo espacial puede ser un condicionamiento, positivo o negativo, del proyecto pedagógico» (Contreras Jordán, 1998, p. 134):

«En la segunda visita al centro, antes de comenzar el periodo de prácticas, mi tutora nos enseñó las instalaciones donde lleva a cabo las sesiones de Educación Física. Conocerlas de antemano me sirvió para planificar unas propuestas u otras en función de lo que allíme encontré. Parecía que el gimnasio ofrecía muchas posibilidades y no tenía la impresión de que fuera a sentirme condicionado por sus limitaciones. Imagino que es la ventaja de hacer las prácticas en uno de los centros públicos más grandes de la provincia» (Diario de prácticas. Febrero de 2005).

"Voy aprendiendo que trabajar en un espacio u otro tiene sus ventajas y sus inconvenientes. Cuando tengo oportunidad de impartir la lección en el pabellón deportivo, condicionantes como las medidas de la sala o los agrupamientos que han de hacer los escolares apenas me preocupan, a diferencia de lo que pasa cuando trabajo en el gimnasio del colegio. Por el contrario, el traslado desde el centro hasta al pabe- 
llón supone una inversión de tiempo que hace que no me permita trabajar todos los contenidos que llevo preparados» (Diario de prácticas. Febrero de 2005).

Ya como profesor, preocupado por el modo en que el recinto pudiera supeditar su práctica, lo primero que hace al acudir a un nuevo centro de trabajo es analizar minuciosamente el estado de las instalaciones en las que iba a ejercer su labor. En Educación Primaria ésta fue su primera actuación como profesor interino:

«El pabellón me ha resultado pequeño, pero no creo que condicione el desarrollo del currículum establecido para la Educación Primaria y menos con el número tan escaso de alumnos por ciclo. Del patio me ha gustado la ausencia de elementos que «deportivicen» la asignatura: no hay líneas de campos pintadas, ni porterías, ni canastas. Desde el punto de vista negativo, en los dos lugares me he encontrado con varios focos que pueden suponer riesgos a la hora de llevar a cabo las actividades. He revisado las espalderas y he quitado los barrotes que estaban en mal estado, he ordenado los materiales para evitar accidentes. Las zonas más peligrosas las he delimitado con bancos, y como eso me quita espacio creo que aprovecharé para hacer ahí las reflexiones» (Diario del profesor. Septiembre de 2009).

Situación que repetiría el siguiente curso escolar, esta vez como profesor interino de Enseñanza Secundaria:

«He ido a ver cómo es el pabellón, ya que es allí donde voy a trabajar casi todas las sesiones. Es muy grande y está cerca del instituto, calculo que tardaremos como mucho tres minutos en ir hasta allí. Visto cómo están los patios y los campos de fuera, me temo que casi siempre vamos a dar la clase a cubierto. A pesar de ser una sala muy grande tiene buena acústica. Además, los materiales se guardan en un almacén, por lo que la sala puede permanecer limpia y ordenada para trabajar. El suelo es de goma, y observo que puede suponer un riesgo porque está deteriorado en algunas esquinas, de manera que procuraré no trabajar mucho en estas franjas» (Diario del profesor. Septiembre de 2010).

Construcción de un ambiente seguro sobre el que desarrollar la práctica pedagógica

Actuar como un mediador que procura dotar de autonomía al alumnado se ve mermado cuando se trabajan contenidos que conllevan riesgo de daño o lesión en el alumnado. Ciertos aprendizajes se ven supeditados por este temor y condicionan el modo de abordar la docencia, que surgen también de ciertas experiencias, como se refleja en su etapa como maestro de Educación Primaria:

«Hoy hemos estado trabajando el tema de la resistencia aeróbica con bancos suecos. A mitad de la sesión pedía los alumnos que colocaran los bancos en mitad de la sala. Uno de éstos se cayó encima del dedo de un alumno y enseguida se puso a gritar y llorar. Fui corriendo, le vendé el dedo como pude y lavamos la herida. Lo cierto es que al niño le ha tenido que doler muchísimo lo que pasó, y yo he sufrido al oírle llamar a su madre y con unos lagrimones como mares. He dedicado el resto de la sesión a explicar la importancia de hacer todo lo posible por garantizar no sólo nuestra seguridad, sino también la de nuestros compañeros. En cuanto a mí, debí haber anticipado el peligro que tiene la utilización de los bancos y los riesgos que implican. Debo estar más atento y saber prever estas situaciones para evitar disgustos en el futuro. Voy a modificar la sesión para no tener que emplear este tipo de materiales» (Diario del profesor. Octubre de 2009).

"He diseñado un circuito de habilidades para el alumnado de primer ciclo con varias estaciones. Algunas de ellas me parecían algo peligrosas para los niños, fundamentalmente porque resultan atractivas y es probable que pierdan el control al realizarlas. Aunque hubiera sido más enriquecedor, desde el punto de vista motriz, realizarlas todas ellas e introducir estaciones cada vez más complejas, me ha dado miedo que alguno de ellos pudiera hacerse daño» (Diario del profesor. Diciembre de 2009).

En su escala de valores como educador está, en primer lugar, la búsqueda del bienestar del alumnado, lo que le lleva incluso a prescindir de determinados contenidos de la materia por considerar que llevan asociados más riesgos que beneficios. De esta manera lo refleja en su diario de profesor de Enseñanza Secundaria:

«En la programación que presentamos mi jefa de departamento y yo a la Inspección Educativa viene reflejado que vamos a trabajar contenidos de baloncesto. No obstante, visto el estado de las pistas del patio, es mejor que lo cambie por algún otro tipo de deporte. En cuanto a las canastas del pabellón, ahí ni me planteo trabajar: están ligeramente ancladas al suelo por unas piedras y no sería la primera vez que algún chico muere por caérsele encima un armatoste de éstos. Cuando les explico que no vamos a trabajar baloncesto son varios los que se quejan. Les explico los motivos y parece que los entienden. Termino la charla poniéndome muy serio y diciendo que me da igual, como profesor, que aprendan o no algo de Educación Física, que sean disciplinados o no, que se diviertan o no; lo que más me preocupa es terminar el curso igual que lo empezamos: sin accidentes de los que lamentarnos» (Diario del profesor. Enero de 2011).

La influencia del diseño y cuidado de las infraestructuras en el desarrollo de la Educación Física

Algunos de los condicionantes espaciales a la hora de abordar la enseñanza son fácilmente modificables, pues dependen fundamentalmente del docente. Otros, no obstante, sería muy complejo transformarlos, pues forman parte de la infraestructura de la instalación, suponen un patrón de influencia crítica relacionado con los entornos de práctica (Day \& Gu, 2012). La solución, en el segundo caso, pasa por tratar de ver en lo que se nos brinda oportunidades de ejercer nuestra labor, estableciendo las tácticas pertinentes para aumentar las posibilidades que ofrecen, algo que busca en su periodo de maestro de Educación Primaria:

"Ya son varias las veces que ha hablado la directora con el encargado del Ayuntamiento para que ponga unas cuerdas alrededor de las colchonetas que cubren los radiadores. Son zonas que suponen un peligro para los escolares y, aunque antes de cada clase las coloco bien, en ocasiones se caen y suponen un riesgo añadido. Desde el Ayuntamiento siempre dan largas, nunca hay tiempo para hacerlo y, lo que es peor, para poder hacerlo yo, necesito su permiso. Como no quiero estar así todo el curso, al final he optado por comprar yo mismo unas gomas elásticas que se pueden quitar fácilmente y que cumplen la función de cubrir los radiadores al tiempo que las colchonetas no se nos puedan caer. No creo que esto forme parte de mis responsabilidades $y$ así me lo confirma el hecho de que necesite autorización, pero así me ahorro disgustos» (Diario del profesor. Noviembre de 2009).

La suciedad es otro aspecto que limitan su desempeño profesional y que condicionan el proceso de enseñanza/aprendizaje:

«El pabellón cada vez está más sucio. Es raro el día que no veo varias cucarachas y arañas por la sala. Hoy, sin ir más lejos, había una araña enorme, que ha armado un buen revuelo desde el principio que ha hecho que me fuera difícil retomar el hilo de lo que pretendía trabajar. Coloqué unas colchonetas a modo de cerco para evitar que saliera y continuédando la clase lo mejor que pude. Pero lo cierto es que, tanto los niños como yo, hemos estado más pendientes de controlar dónde estaba que de la clase. [...]. Como entiendo que esta situación es intolerable, al final del día le pido permiso a la directora para redactar un escrito dirigido al Ayuntamiento para que tome las medidas oportunas. ¿Tantas cosas tienen que hacer los que trabajan allípara dejar de lado todo lo que concierne al colegio?, ¿o simplemente es una cuestión de desinterés? Me pregunto si la situación sería la misma si el alcalde tuviera matriculados a sus hijos en este colegio o, de ser así, contaría con unas instalaciones impecables» (Diario del profesor. Diciembre de 2009).

«Cada día acudo al centro con la incertidumbre de cuántos bichos me voy a encontrar. Así es complicado trabajar, ir con ganas y decidido a enseñar. He tenido que barrer la sala porque había un pájaro muerto que a saber desde cuándo estaba. Limpiar la sala ya sí que es algo fuera de mis responsabilidades y, aunque lo haría por intentar garantizar unas condiciones más salubres para el alumnado, me puede mi fobia con las arañas. Estoy deseando que llegue cualquier atisbo de buen 
tiempo para estar todo el día en el patio» (Diario del profesor. Diciembre de 2009).

Se comprueba, de esta manera, el modo en que el contexto influye en el trabajo y la práctica pedagógica pues, como afirma McLaughlin:

«El carácter y la calidad de la enseñanza escolar que encontramos en cualquier aula un día cualquiera indican mucho más que los atributos, la energía y la pericia de un profesor. Los docentes llevan a sus aulas las condiciones de sus múltiples contextos, como marcos normativos, apoyos concretos, limitaciones percibidas o reales para la construcción de la práctica»(McLaughlin, 2005, en Day \& Gu, 2012,p. 68).

La influencia de los espacios y las infraestructuras en el desarrollo de la identidad profesional docente

Querer transformar los lugares de práctica de modo que se pueda crear en ellos una «atmósfera» (Van Manen, 2008, p. 73) en la que los estudiantes se sientan protegidos, confiados y dispuestos para el aprendizaje, exige una reflexión docente de los criterios que pretende alcanzar con su enseñanza para no incurrir en actuaciones que la dificulten. De esta manera, el educador procura hacer del espacio un lugar más agradable para el aprendizaje del alumnado de Educación Primaria, olvidando que con ello puede estar provocando situaciones no previstas:

«He ordenado la sala y he procurado hacerla más acogedora, más nuestra. He puesto algunos mapas del mundo para cuando trabajemos juegos de otros países, la he decorado con algunos de los dibujos que vamos haciendo en Educación Artística, etc. También he puesto algunos materiales a la vista de los escolares, como aros, frisbees, indiacas, pensando que eso quizá les motivaría. No he sido consciente, hasta finalizar la clase con los pequeños, de lo atractivo que les resultan estos materiales. En cuanto los han visto se han subido a las espalderas y a las colchonetas para hacerse con ellos. Ha sido un error por mi parte que debería haber previsto porque podría haber habido algún accidente» (Diario del profesor. Octubre de 2009).

No se trata únicamente de cumplir los requisitos básicos de higiene y seguridad de los espacios de aprendizaje; se trata, sobre todo, de procurar un ambiente idóneo que facilite el desarrollo de la materia y de las relaciones personales que en ella tienen lugar. Ahora bien, durante su etapa de Enseñanza Secundaria, nuevamente es consciente de que la creación de estas condiciones favorables, por sencillo que pudiera parecer, acaba siendo una tarea titánica:

«El ambiente de trabajo condiciona mucho mi manera de enfocar la docencia, pero también me doy cuenta de que condiciona las conductas de los estudiantes. En el pabellón hace muchísimo frío y desde el Ayuntamiento me ponen pegas a la hora de dar la calefacción: cuando no es por temas de dinero es porque se estropea la calderay, cuando no, es alguna otra cosa. En cuanto llegan los chicos a la sala se ponen a correr y a jugar para entrar en calor, y me cuesta muchísimo poder comenzar la clase. En los días de más frío, he optado por no meter apenas parones ni momentos de reflexión para evitar que se queden fríos» (Diario del profesor. Diciembre de 2010).

El deterioro de los espacios dificulta no solo la labor profesional, sino que puede llevar a cierto desencanto con la profesión escogida:

«Escucho a menudo, no sólo en la prensa sino de boca de mis compañeros, que la actual crisis económica está haciendo mella en el sistema educativo. No me hace falta escucharlo de nadie, en mi día a día me voy dando cuenta de ello. Aunque a todos los políticos se les llena la boca diciendo que en cuestiones de educación no van a escatimar en gastos, la verdad es que basta echar un vistazo a los lugares donde debo dar clase para entender que no es cierto. Y no me refiero a que tenga más o menos recursos que, por cierto, por entender precisamente la situación por la que pasamos no he solicitadoningún tipo de material al centro. Se trata más bien de los problemas de conservación del pabellón y del gimnasio, donde cada día hay más goteras, y del frío que hace porque no es posible encender la calefacción. Esta situación exige mucho de mí, ya no sé cómo hacer para modificar la práctica docente para hacer frente a estas trabas. Soy consciente de que esta situación me está haciendo perder parte de mi ilusión por ser docente, y esto me preocupa» (Diario del profesor. Enero de 2011).
Pese a que el educador considera que, en general, la situación de los espacios donde se desarrolla la asignatura de Educación Física ha mejorado, en los centros en los que ha desarrollado su labor éstos no proporcionan una sensación de seguridad ni de bienestar al discente. Más aun, la dimensión pedagógica de la enseñanza se ha visto acotada por el lugar de enseñanza. Van Manen (2008, p. 78) entiende que «los materiales expuestos y el mobiliario del aula también pueden servir para reinterpretar la importancia de aprendizajes anteriores». Así, algunos de los espacios en los que ha trabajado han condicionado los contenidos a enseñar eincluso la orientación que habría de darle a la docencia, como le sucede en Enseñanza Secundaria:

«Aunque solamente tengo una sesión a la semana en la que utilizo el gimnasio, cada vez que vamos allí los alumnos dan por supuesto que vamos a trabajar contenidos de habilidades gimnásticas. Saben de sobra que si vamos es porque el pabellón lo va a utilizar la otra profesora,pero todos los días me dicen que entonces para quéestán allí todos esos aparatos. Y tienen razón. Además de lo mucho que ocupan y de los riesgos que supone que estén allí, cada vez que los ven se les vienen a la cabeza las reminiscencias de la antigua gimnasia» (Diario del profesor. Abril de 2011).

\section{Discusión}

Tal y como se ha reflejado en la autoetnografía, adaptar los espacios y los contextos en que se desarrollan las lecciones de Educación Física con la intención de favorecer los aprendizajes y reducir las contingencias es una de las principales preocupaciones del docente novel (Brooks \& Thompson, 2015). En este sentido, el docente se considera responsable de crear un entorno educativo seguro y contar con la disposición apropiada para reducir las posibilidades de accidente en los escolares. Es, la práctica reflexiva del educador, lo que le permite una mayor comprensión de la centralidad de los espacios y los materiales en el pensamiento y práctica docente del caso presentado aquí (González Calvo \& Barba, 2014; Sá, 2002).

Los espacios tienen un vínculo especial con la Educación Física. Para comenzar, es una de las pocas asignaturas que implica abandonar las aulas donde se imparten el resto de las asignaturas. Los espacios donde se imparten las clases de Educación Física en la España contemporánea están ligados al imaginario extraescolar (fundamentalmente deportivo), lo que explica una serie de características que colisionan con la lógica pedagógica. Suele tratarse de un espacio aséptico, sin marcas de apropiación de los grupos que los habitan o los docentes que se encargan del área. Por poner una metáfora, se entiende el gimnasio más como una habitación de hotel (que puede ser usada por múltiples huéspedes sin ser propiedad de nadie) que como un cuarto propio, por lo que la lógica que predomina en su diseño, mantenimiento y uso no es necesariamente, ni de forma predominante, la pedagógica (Öhman \& Quennerstedt, 2017).

Esto hace que el docente, mucho más si es novel, deba acomodar su práctica a los otros usos. Si un docente tiene una historia y peso en el centro escolar, si ha sido capaz de establecer relaciones y alianzas que permitan inclinar el espacio hacia su vertiente más escolar, es posible transformar los espacios, hacerlos más congruentes con un entorno educativo. Pero si no es así, la sensación que tiene el novel es ir a remolque. Y en no pocos casos, por ejemplo cuando ve desidia en elementos como la limpieza o la temperatura, los entiende también como una muestra más de minusvaloración delárea.

La transformación y mantenimiento en condiciones óptimas de los espacios requieren tiempo y socios poderosos, lo que, en principio, choca con la provisionalidad y el bajo estatus de los docentes noveles en particular, y de la Educación Física en general (González Calvo \& Barba, 2013). A no ser que el lugar (o lugares) donde se imparte la Educación Física sean asumidos por toda la comunidad escolar como responsabilidad de todo el centro (y no solo delárea de Educación Física y de quienes la imparten) estamos condenados a que no sean una prioridad educativa. La realidad en nuestro contexto es que, en muchos casos, noveles (y veteranos) ven en los espacios un amplificador del 
escaso estatus del área y de su reducido poder para cambiar las cosas: gimnasios sucios, fuertemente influidos por las actividades extraescolares que acogen, ocupados durante ciertos momentos del curso por otras actividades del colegio - festivales, representaciones...--, con almacenes descuidados y poco accesibles, con mala sonoridad y aislamiento térmico... Los espacios, en definitiva, muestran la escasa consideración que el sistema educativo en su conjunto muestra por el área de Educación Física, pues afecta seriamente a las propuestas educativas y también a la salud de quienes las usan: alumnado y profesorado.

En estas condiciones, que se dan en el caso que presentamos aquí, no queda otra que acudir, con una cierta aprensión, a ver 'lo que ha tocado', y asumir las dos tareas que le quedan por delante: tratar de acomodar el espacio lo que buenamente se pueda (identificar posibles riesgos, descartar algunos materiales y elementos o recuperar otros de almacén) y reformular algunos contenidos de la programación o aspectos metodológicos para ajustarla al espacio disponible.

Esto es lo que vemos desde su primer diario, como estudiante en prácticas, y que se repite cada inicio de curso como docente. Es lo que vemos también en los estudiantes en prácticas en sus primeros acercamientos a los centros en los que realizarán su prácticum. Uno de los ritos que les calma su ansiedad es la visita que sus tutores suelen hacerles por las instalaciones del colegio. El espacio (junto, quizá, los horarios y los escolares a su cargo) concreta su estancia en los centros y les pone en situación de pensar más claramente en una programación, de cómo éste les influye y les condiciona su práctica pedagógica y el modo en que abordan los procesos de enseñanza/aprendizaje de la asignatura.

\section{Conclusiones}

Las condiciones laborales de los noveles conllevan cambios que inciden demaneraparticular en la Educación Física(Richards \& Padaruth, 2017). Si la programación depende, en buena medida, de las instalaciones y del trabajo previo que se debe hacer con ellas, ser novel de Educación Física es un poco más complicado que serlo de otras áreas en las que el desarrollo de la materia está menos condicionado por la infraestructura (aunque siempre sea un factor importante). Las instalaciones, según vemos en las diferentes viñetas presentadas en este artículo, influyen, sobre todo, en los contenidos que imparte (hay algunos que requieren instalaciones específicas-pensemos en natación-, pero incluso los que no son tan dependientes de algo específico, están mediados por el espacio o las condiciones de seguridad o de uso que reclaman). También hay una influencia notable en las metodologías que se pueden emplear (hace sobre todo énfasis en la autonomía que considera que puede otorgar para no sentir que hace dejación de responsabilidades). E docente asume como parte de la preparación del curso, la revisión y acondicionamiento de los espacios en los que impartirá las clases. Asimismo, de la autoetnografía se intuye una sensación de que el sistema (social y escolar) no se preocupa lo debido por la Educación Física, y que mantiene a esta condicionada por unas imágenes extraescolares que no cambiarán sin un fuerte apoyo externo (la Educación Física, en esta imagen dominante que el espacio refleja y perpetúa, es fundamentalmente actividades de juego y/o diversión, es postergada frente a otras prioridades escolares o extraescolares y, en la actualidad, está vinculada casi exclusivamente con aspectos higiénicos y de prevención de la obesidad entre el alumnado) (del Valle, Ruiz, De la Vega, Velázquez, \& Díaz, 2005; Fraile Aranda, 2004; Molina, Peiró, \& Devís, 2004).

Por otra parte, el hecho de que las instalaciones para el área de Educación Física sean frecuentemente percibidas como algo que no es solo (ni predominantemente) escolar, supone un recuerdo constante de su carácter educativo periférico, incluso de manera literal. Hay que salir del recinto escolar o del edificio donde se desarrollan la mayoría de las otras actividades, siendo ese traslado visto como una responsabilidad del área y docente de Educación Física en su mayor parte. El docente comienza su carrera enfrentándose en solitario al reto de tener unas instalaciones adecuadas, algo que, claramente, escapa de las posibilidades de su posición laboral. Esta situación condiciona, en gran medida, su práctica pedagógica. Mención especial merece el riesgo (real o percibi- do) que tiene el docente a que el alumnado pueda tener un accidente 0 una lesión, aspecto permanente en su tiempo pedagógico y en su programación educativa. La salud e integridad de los escolares se ve como una responsabilidad personal (Olley, Cohn, \& Cowan, 2010), con un escaso apoyo y comprensión por parte de las familias o resto del sistema en el caso de que haya un 'accidente' (no hablamos de negligencia), lo que limita las propuestas y el grado de cesión de autonomía al alumnado. De acuerdo a los escritos del diario, el sistema escolar no es visto como un aliado y, comprensiblemente, el docente puede anteponer los aspectos más básicos para la seguridad de sus estudiantes y suya propia (no lesionarse, no ser demandado) a los aspectos más enriquecedores que podrían alcanzarse (aprender a valorar y gestionar el riesgo, transferir autonomía) (Chappell, 2006). Y es que hay elementos que el docente tendría en cuenta pero, aparentemente, los encargados de diseñar y mantener las instalaciones no tanto: la acústica, la temperatura (a veces, acústica y temperatura se contraponen, como en los gimnasios calentados con aerogeneradores), la limpieza, la posibilidad de que el almacén sea accesible y utilizable por parte del alumnado, la iluminación, el tipo de suelo, elementos que amueblan las salas... Esto nos puede llevar a la pregunta de quién asesora en el diseño y dotación de los espacios para la Educación Física, y cuáles son los criterios que se priorizan (Hill \& Hulbert, 2007).

Entre las viñetas seleccionadas hemos visto también algún aspecto que habla de las fobias y filias personales. Una escena recoge la presencia de arañas que, según valoramos, no debe ser entendida tanto como una crítica a la limpieza (sería irreal pretender un espacio aséptico) sino como un ejemplo más de la naturaleza corporal dela enseñanza(Martínez Álvarez y González Calvo, 2016). Este docente, como cada uno de nosotros, está conformado por una historia personal grabada en su cuerpo, que marca el rango de experiencias que somos más proclives a afrontar y el significado que damos a lo que vivimos. Aunque en este estudio han aparecido solo espacios cerrados, nos lleva también a pensar sobre cómo cada cual ha incorporado una cierta propensión o rechazo hacia espacios naturales y que podría también suponer de hecho un mayor o menor empeño en incluir estos espacios como lugares propios de la Educación Física en función de si tenemos, o no, tolerancia a las inclemencias, a los terrenos abruptos, al contacto con animales, a la incertidumbre... Aunque no siempre es fácil de cambiar, es necesario al menos reconocer las huellas corporales desde las que reinterpretamos la experiencia vivida y, por tanto, tendemos o no a incluir como parte de las posibilidades ofrecidas al alumnado. En los escritos autobiográficos analizados se reflejan también otros aspectos culturales e históricos en el que se desarrollan (la España interior de la primera década del siglo). Vemos, en la viñeta de su prácticum, cómo su colegio complementaba sus instalaciones precarias dentro del recinto escolar, con unas instalaciones más modernas, cuidadas y dotadas fuera del colegio. Las instalaciones que muchos colegios deben emplear al ser las suyas insuficientes o inadecuadas tienen una lógica que se separa todavía más de la lógica pedagógica, ya que están pensadas y orientadas para las actividades deportivas y de condición física para las que fueron diseñadas. Más todavía que en las instalaciones escolares, los pabellones deportivos a los que acude están fuera de su control y su presencia en ellos es de mero usufructuario.

Alguna de las implicaciones de esta investigación derivan en la necesidad de abordar en la formación inicial la dimensión subjetiva de las posibilidades percibidas por los docentes en los espacios y los materiales a raíz de su biografía y su formación, así como considerar la dimensión colectiva que entraña el cambio de los usos y diseños de infraestructuras, para tratar de superar la visión individual desde la que se afronta la docencia.

Sería conveniente contar con más relatos autoetnográficos de docentes de diferentes características (género, edad, planteamientos pedagógicos...) y en diversos momentos profesionales para conocer cómo se dan las interacciones entre recursos, formación y biografía que han aparecido en el presente estudio en un conjunto mucho más amplio de docentes de Educación Física. 


\section{Referencias}

Blández Ángel, J. (1995). La utilización del material y del espacio en Educación Física. Propuestas y recursos didácticos. Barcelona: Inde.

Brooks, C., \& Thompson, M. D. (2015). Insideness and Outsideness: An Autoethnography of a Primary Physical Education Specialist Teacher. European Physical Education Review, 21(3), 325-229.

Bruner, J. (1990). Acts of meaning: Four lectures on mind and culture (Jerusalem-Harvard Lectures). Cambridge, MA: Harvard University Press.

Contreras Jordán, O. (1998). Didáctica de la Educación Física: un enfoque constructivista. Barcelona: Inde.

Chang, H. (2013). Individual and Collaborative Authoetnography as Method. A Social Scientist's Perspective. In S. Holman Jones, T. E. Adams, \& C. Ellis (Eds.), Handbook of Autoethnography (pp. 107-122). Walnut Creek, CA: Left Coast Press.

Chappell, A. (2006). Safe practice, risk assessment and risk management. In S. Capel, P. Breckon, \& J. O’Neill (Eds.), A practical guide to teaching Physical Education in the Secondary School (pp. 100118). New York: Taylor \& Francis.

Chong, S., Low, E. L., \& Goh, K. C. (2011). Emerging Professional Teacher Identity of Pre-Service Teachers. Australian Journal of Teacher Education, 36(8), 50-64.

Day, C., \& Gu, Q. (2012). Profesores: vidas nuevas, verdades antiguas. Madrid: Narcea.

De Fina, A., \& Georgakopoulou, A. (2008). Analysing narratives as practices. Qualitative Research, 8, 379-387.

del Valle, S., Ruiz, L. M., De la Vega, R., Velázquez, R., \& Díaz, P. (2005). ¿ ¿Sabemos qué contenidos aprenden los escolares en Educación Física? Motricidad. European Journal of Human Movement, 13, 87 106.

Delle Fave, A. (2009). Optimal experience and meaning: Which relationship. Psychological Topics, 18(2), 285-302.

Denzin, N. K. (1989). Interpretive biography. London: Sage.

Dervent, F. (2015). The Effect of Reflective Thinking on the Teaching Practices of Preservice Physical Education Teachers. Issues in Educational Research, 25(3), 260-275.

Fraile Aranda, A. (2004). Modelos y tradiciones en la formación del profesorado de Educación Física. In A. Fraile Aranda (Ed.), Didáctica de la Educación Física: una perspectiva crítica y transversal (pp. 291-314). Madrid: Biblioteca Nueva.

Garret, R., \& Wrench, A. (2014). «If They Can Say It They Can Write It»: Inclusive Pedagogies for Senior Secondary Physical Education. International Journal of Inclusive Education, 20(5), 486-502.

Gergen, K. (1997). Realities and relationships: Soundings in social construction. Cambridge: Harvard University Press.

Goffman, E. (1981). La presentación de la persona en la vida cotidiana [Introducing the person to everyday life]. Buenos Aires: Amorrortu.

González-Calvo, G., \& Arias-Carballal, M. (2017). Effects from audit culture and neoliberalism on university teaching: an autoethnographic perspective. Ethnography and Education, 1-15. doi:10.1080/ 17457823.2017.1347885

González Calvo, G. (2013a). Evolución de la identidad profesional de un docente novel de Educación Física: análisis a partir de la reflexión de sus experiencias personales y de su propia práctica. Tesis Doctoral. Universidad de Valladolid, Valladolid.

González Calvo, G. (2013b). La lucha de un docente novel de Educación Física por el reconocimiento de su profesión narrada desde una perspectiva autobiográfica. Ágora para la Educación Física y el Deporte, 15(2), 80-102.

González Calvo, G., \& Barba, J. J. (2013). La perspectiva autobiográfica de un docente novel sobre los aprendizajes de Educación Física en diferentes niveles educativos. Cultura, Ciencia y Deporte, 8(24), 171-181.

González Calvo, G., \& Barba, J. J. (2014). Formación permanente y desarrollo de la identidad reflexiva del profesorado desde las perspectivas grupal e individual. Profesorado: Revista de Currículum y Formación del Profesorado, 18(1), 398-412.

González Calvo, G, Barbero González, J. I., Bores Calle, N., \& Martínez Álvarez, L. (2014). (Re)construction of a teacher's professional identity from his initial training: Autobiographical narration. The Open Sports Science Journal, 7(2), 113-120.

González Calvo, G., \& Becerril González, R. (2013). El recorrido investigador de un educador novel explicado desde una perspectiva autobiográfica. Revista Electrónica Interuniversitaria de Formación del Profesorado, 16(3), 61-78.
González Calvo, G., \& Martínez Álvarez, L. (2009). Aproximación a los significados e interpretaciones de la lesión en futuros docentes de Educación Física por medio de narraciones autobiográficas. Retos: Nuevas Tendencias en Educación Física, Deporte y Recreación, 15(46), 35-40.

Guba, E., \& Lincoln, Y. S. (2005). Paradigmatic controversies, contradictions, and emerging confluence. In N. K. Denzin \& Y. S. Lincoln (Eds.), The Sage Handbook of Qualitative Research (pp. 191-215). Thousand Oaks: Sage.

Gubrium, J. F., \& Holstein, J. A. (1998). Narrative practice and the coherence of personal stories. The Sociological Quarterly, 39, 163-187.

Gubrium, J. F., \& Holstein, J. A. (2009). Analyzing narrative reality. Thousand Oaks: Sage.

Hill, G., \& Hulbert, G. (2007). Using a Physical Education Environmental Survey to Identify Areas of Concern and Improve Conditions. Strategies: A Journal for Physical and Sport Educators, 21(1), 21-25.

Holman Jones, H., Adams, T., \& Ellis, C. (2013). Handbook of autoethnography. Walnut Creek: Left Coast Press.

Lieblich, A., Tuval-Mashiach, R., \& Zilber, T. (1998). Narrative research: Reading, analysis and interpretation. London: Sage.

Liston, D. P., \& Zeichner, K. M. (2003). Formación del profesorado y condiciones sociales de la escolarización. Madrid: Morata.

Lund, J. (2013). Activity in Physical Education: Process or Product? Journal of Physical Education, Recreation \& Dance, 84(7), 16-17. doi:10.1080/07303084.2013.817897

Martínez Álvarez, L. y González Calvo, G. (2016) Docentes de carne y hueso: enseñar con cuerpo. Ágora para la EF y el deporte, 18(3), 259275. Retrieved from http://agora-revista.blogs.uva.es/files/2016/12/ agora_18_3d_martinez_et_gonzalez.pdf

MacPhail, A., \& Halbert, J. (2010). 'We had to do intelligent thinking during recent PE': students' and teachers' experiences of assessment for learning in post primary physical education. Assessment in Education: Principles, Policy \& Practice, 17(1), 23-39.

McCoy, L., Esslinger, K., \& Baghurst, T. (2017). Injury and Inclusion: Understanding Common Legal Concerns in Physical Education. Strategies: A Journal for Physical and Sport Educators, 30(5), 3-11.

Merrie, M., Shewmake, C., \& Calleja, P. (2016). Injury Prevention in Physical Education: Scenarios and Solutions. Strategies: A Journal for Physical and Sport Educators, 29(4), 15-18.

Molina, J. P., Peiró, C., \& Devís, J. (2004). Un estudio sobre los materiales curriculares impresos en Educación Física: implicaciones para la formación del profesorado. Movimiento, 10(1), 41-70.

Öhman, M., \& Quennerstedt, A. (2017). Questioning the no-touch discourse in physical education from a children's rights perspective. Sport, Education and Society, 22(3), 305-320. doi:10.1080/ 13573322.2015.1030384

Olley, R. I., Cohn, A., \& Cowan, K. C. (2010). Promoting safe schools and academic success: Moving your school from punitive discipline to effective discipline. Communique, 39(1), 7-8.

Pérez-Samaniego, V. M., Devís-Devís, J., Smith, B. M., \& Sparkes, A. C. (2011). La investigación narrativa en la educación física y el deporte: qué es y para qué sirve. Movimento, 17(1), 11-38.

Richards, K. A., \& Padaruth, S. (2017). Motivations for Pursuing a Career in Physical Education: The Rise of a Fitness Orientation. Journal of Physical Education, Recreation \& Dance, 88(4), 40-46.

Riessman, C. K. (2008). Narrative methods for human sciences. Thousand Oaks: Sage.

Sá, J. (2002). Diary Writing: An Interpretative Research Method of Teaching and Learning. Educational Research and Evaluation, 8(2), 149-168. doi:10.1076/edre.8.2.149.3858

Shenton, A. K. (2004). Strategies for ensuring trustworthiness in qualitative research projects. Education for Communication, 22, 63-75.

Smith, B., \& Sparkes, A. C. (2008). Contrasting perspectives on narrating selves and identities: an invitation to dialogue. Qualitative Research, 8(1), 5-35.

Stolz, S. A., \& Kirk, D. (2015). David Kirk on physical education and sport pedagogy: in dialogue with Steven Stolz (part 1). Asia-Pacific Journal of Health, Sport and Physical Education, 6(1), 77-91. doi:10.1080/18377122.2014.997862

Surbeck, E., Han, E., \& Moyer, J. (1991). Assessing reflective responses in journals. Educational Leadership, 48(6), 25-27.

Van Manen, M. (2008). El tono en la enseñanza. El lenguaje de la Pedagogía. Barcelona: Paidós.

Zabalza Beraza, M. A. (2008). Diarios de clase: un instrumento de investigación y desarrollo profesional. Madrid: Narcea. 